\title{
The Effect of Carbon Dioxide Laser on Aphthous stomatitis Treatment: A Double-Blind Randomized Clinical Trial
}

\author{
${ }^{1}$ Faculty of Dentistry, Urmia University of Medical Sciences, Urmia, Iran \\ ${ }^{2}$ Implantology, Urmia University of Medical Sciences, Urmia, Iran \\ ${ }^{3}$ Faculty of Dentistry, AJA University of Medical Sciences, Tehran, Iran \\ ${ }^{4}$ Urmia University of Medical Sciences, Urmia, Iran \\ ${ }^{5}$ School of Dentistry, Tehran University of Medical Sciences, Tehran, Iran \\ ${ }^{6}$ Health Promotion, Oklahoma State University, Stillwater, OK, USA
}

Seyyed Amir Seyyedi', Pooya Olyaee ${ }^{2}$, Reza Fekrazad ${ }^{3}$, Sanaz Partovi ${ }^{4}$, Maryam Baghizadeh Fini ${ }^{5,6^{*}}$ (I)

\section{*Correspondence to \\ Maryam Baghizadeh Fini;DDS, \\ School of Dentistry, Tehran \\ University of Medical Sciences, \\ Tehran, Iran. \\ Master's Student, Master of \\ Health Promotion, Oklahoma \\ State University, Stillwater, OK \\ USA. Tel: +1 (405) 612-1737 \\ Email: m.baghizadeh92@gmail. com}

Published online December 30 2020

\begin{abstract}
Introduction: This randomized clinical trial was designed to investigate the effect of carbon dioxide laser on the treatment of minor recurrent aphthous stomatitis (miRAS) as a painful and common oral ulcer.

Methods: Fifteen patients with miRAS entered into this study after compliance with the inclusion and exclusion criteria. The patients were assigned to three groups of pulsed $\mathrm{CO}_{2}$ laser, continuous $\mathrm{CO}_{2}$ laser, and control. A pulsed $\mathrm{CO}_{2}$ laser with power of $261 \mathrm{~W}$ and pulse duration of 180 microseconds and continuous $\mathrm{CO}_{2}$ lasers with the power of $1 \mathrm{~W}$ and radiation duration of 5-10 seconds were used. In the control group, topical triamcinolone ointment of $0.1 \%$ was used. The degree of pain was measured using the visual analogue scale (VAS). The patients were evaluated in terms of pain intensity before and immediately after the treatment and at follow-up hours, 12, 24, 48, and 72 hours after the intervention, and changes in the size of the ulcers were measured before the treatment and on third, fifth, and seventh days.

Results: The effect of $\mathrm{CO}_{2}$ laser with pulsed and continuous radiation on the pain intensity of aphthous lesions, immediately after the treatment and at subsequent follow-up hours were similar and even better than the control group $(P<0.05)$. All three treatments had a similar effect on the size of the ulcer, which was not statistically significant $(P>0.05)$. Conclusion: The results of this randomized controlled trial (RCT) showed that the carbon dioxide laser, whether with pulsed radiation or continuous radiation compared to the control group, had a better impact on the pain relief of aphthous lesions.

Keywords: Recurrent aphthous stomatitis; Laser; Carbon dioxide lasers; Pain.
\end{abstract}

\section{Introduction}

Recurrent aphthous stomatitis (RAS) is a common and recurrent disease diagnosed with recurrent ulcers limited to oral mucosa in patients who have no other signs of systemic disease. ${ }^{1,2}$ RASs are painful, round, or elliptical ulcers recurring at different intervals. These ulcers include a central necrotic part with fibrinous and whitish gray exudate and a red halo is around it. ${ }^{1,3,4}$

Recurrent aphthae are observed in three forms of minor, major, and herpetic aphthae. ${ }^{1,4,5}$ Minor recurrent aphthae are the most common form (80\%-90\%) of the disease and the patient has a history of former aphthae, ${ }^{2}$ usually healed during 7-10 days without a scar. Their size is 2-4 $\mathrm{mm}$ (less than $1 \mathrm{~cm}$ ) on average and the most prevalent place of involvement is buccal and labial mucosa. ${ }^{6,7}$ Aphthous stomatitis is the most common ulcerative disease of the oral mucosa. ${ }^{8,9}$ The prevalence reported in the normal population in the world is between $5 \%$ and $66 \%$ with the mean of $20 \%{ }^{5}$ and the prevalence is $25.2 \%$ in Iran. ${ }^{10}$ The prevalence of the disease in people with a higher social-economical level is more than others and that amount in females is observed slightly more than in males. ${ }^{3}$ There is no relationship between the pain degree of aphthous lesions and the size of the lesions and it can be very intensive in such a way that it may disrupt one's eating, drinking, and even talking. ${ }^{2,11}$

Because there is no agreement on the cause of the aphtha, it is difficult to find an effective treatment for it. Therefore, three goals are followed in the treatment of recurrent aphthous stomatitis: patient's pain control, accelerated ulcer healing, and reduced recurrence of lesions. Different drugs and treatments are used to reduce

Please cite this article as follows: Seyyedi SA, Olyaee P, Fekrazad R, Partovi S, Baghizadeh Fini M. The Effect of carbon dioxide laser on Aphthous stomatitis treatment: a double-blind randomized clinical trial. J Lasers Med Sci. 2020;11(suppl 1):S67-S72. doi:10.34172/ jlms.2020.S11. 
the pain of aphthous stomatitis, including mouthwashes, antibiotics, topical anesthetics, using silver nitrate pen, and cryotherapy. ${ }^{11-14}$ In most of the patients, the main pivot of the treatment is topical and systemic corticosteroids which have complications such as weakening the immune system and reducing the barrier defenses of the body against a variety of infections, delaying the ulcer healing, reducing and weakening of the mucous membranes of the gastrointestinal system, especially the stomach, which causes and exacerbates the gastrointestinal ulcers. ${ }^{15}$

Another therapeutic option for this complication is laser treatment that seems to be effective in reducing pain intensity, inflammation and edema, accelerating ulcer healing, and improving the depth of the tissues and nerves. These effects in low-power lasers result from the changes that happened due to the laser therapy in the cellular mechanism. ${ }^{16-19}$

Lasers used to treat aphthous lesions include InGaAlP, $\mathrm{Nd}$ : YAG, $\mathrm{CO}_{2}$ and diode lasers ${ }^{20-24}$ among which the $\mathrm{CO}_{2}$ laser is superior to other types of lasers due to its simultaneous effects on pain and aphtha healing time and its very low power with low radiation duration and it can therefore be effective in the treatment of recurrent aphthous stomatitis. ${ }^{20}$ The $\mathrm{CO}_{2}$ laser with a $10600 \mathrm{~nm}$ wavelength is used to treat oral aphthous lesions with different average power of $0.7 \mathrm{~W},{ }^{25} 1 \mathrm{~W}^{2,26} 2 \mathrm{~W}^{23,27}$ and 3 $\mathrm{W}^{28}$ continuously with ${ }^{28,29}$ or without local anesthesia ${ }^{26,30}$ beyond the clear gel. ${ }^{2,26,27}$

The present study was designed and performed to review the effect of a session of using carbon dioxide laser on pain and size of minor aphthous lesions and compare its effects with the control group.

\section{Materials and Methods \\ Study Plan}

This randomized and interventional clinical trial study was done on patients referred to the clinics and offices in Urmia after obtaining permission from the Ethics Committee of Urmia University of Medical Sciences. This clinical trial was registered in the Iranian Registry of Clinical Trials (IRCT) with code IRCT20180224038840N1. Fifteen people who had a clinical diagnosis of minor RAS (miRAS) with a detailed history and examination by a knowledgeable clinician were selected. For all patients, the usual clinical and paraclinical procedures took place to reject the underlying causes. Then, the patients entered into the study after compliance with the inclusion and exclusion criteria. ${ }^{6,724,25}$ The inclusion criteria were:

1. A definitive diagnosis of minor aphthous stomatitis,

2. It should be less than 72 hours after lesions form,

3. The diameter of the lesions is less than $10 \mathrm{~mm}$.

Exclusion criteria include:

1. Underlying systemic problems (such as anemia, diabetes, pregnancy),

2. Atypical cases of aphtha and suspected cases of Behçet's syndrome, inflammatory bowel diseases, etc,
3. Simultaneous use of any type of topical drugs for aphtha treatment,

4. Using any type of topical or systemic anesthetic compounds before laser irradiation, during it and within 4 days in follow-up,

5. Using any kind of systemic treatment of aphtha during three previous months,

6. Age less than 12 years.

Before the treatment, the research nature of the protocol and the implementation of the protocol were described for the patient and informed consent was obtained.

\section{Intervention}

In the first session, the initial examination was performed by a general dentist. In this session, the history of medicine use, previous lesions, and various diseases such as gastrointestinal diseases, blood diseases, and skin diseases was obtained. The size of the lesions was measured and recorded by a periodontal probe. Paraclinical tests including complete blood count (CBC), fasting blood sugar (FBS), ferritin, and thyroid stimulating hormone (TSH) were conducted.

The patients were randomly divided into three groups of 5: patients of the pulsed laser as group A, patients of the continuous laser as group B, and the control group as group $\mathrm{C}$. The randomizing operation was done confidentially and at a site outside the center by the block randomization method.

In the patients of laser groups, before starting laser therapy on aphthous lesions, a layer of $3 \mathrm{~mm}$ thick transparent gel (Lubricating Jelly, Abzar Darman Co., Iran) which contains a high amount of water (87.5\%) without any topical anesthetic property was placed. Before the start of radiation, the patient and staff of the operating room put on safety goggles which are appropriate to the wavelength of the $\mathrm{CO}_{2}$ laser $(10600 \mathrm{~nm})$. For those lesions using the continuous laser, lesions were treated by the laser with a power of $1 \mathrm{~W}$ above the gel layer. The radiation duration was 5 to 10 seconds and the radiation method was spiral. In the type of pulsed radiation, the power of the laser was $261 \mathrm{~W}$, pulse duration was 180 microseconds and the pulses interval was 40 milliseconds. The laser used in the above-mentioned groups was Ultra-Dream Daeshin Enterprise, model: DS-40U type, made in South Korea with a wavelength of $10600 \mathrm{~nm}$ and maximum power of 315 W. Given the presence of laser therapy, there was no need for topical or systemic anesthetics. In the control group, oral ointment of $0.1 \%$ triamcinolone acetonide (Teriadent, Raha Pharmaceutical Co., Iran) was used. This ointment was applied by the patient in the aphthous lesion area using a special daily applicator, four times after meals and before bedtime.

Before, immediately after and 12, 24, 48, and 72 hours after the treatment, pain intensity was evaluated by the patients based on VAS criteria in which score 0 means no pain and 10 means maximum and intolerable pain. In 
the follow-up on the third, fifth, and seventh day after the treatment, due to self-limiting conditions, fatigue, loss, and healing within 10-14 days, the patient was examined at the office by the project executor who was not aware of group types. At these sessions, the size of the aphthous lesions was measured in millimeters with a periodontal probe and was compared to the size of the lesion before the treatment.

Laser therapy was performed for patients by a laser fellowship with the same condition. The amount of pain and size of the lesions after laser treatment was checked by an examiner (general dentist) who was unaware of the type of treatment and the type of grouping. The statistician who performed the analyses of this study was also unaware of the treatments and the type of patients' grouping. Since both the examiner and the statistician were non-informative, our study is a double-blind study.

\section{Statistical Analysis}

Obtained data were analyzed by SPSS 20 software. The results were expressed as mean \pm standard deviation. Statistical evaluation was reviewed using tests of repeated measurement ANOVA and one-way ANOVA. P values less than 0.05 in a two-range test were considered significant.

\section{Results}

Fifteen patients (13 females and 2 males) who had 15 minor aphthous lesions were selected and remained in the plan until the end of the study. The mean age of the patients was $34.54 \pm 14.93$ years (17-60 years old) (Figure 1).

The comparison of pain intensity average before the treatment in the studied groups, including the pulsed carbon dioxide laser, the continuous carbon dioxide

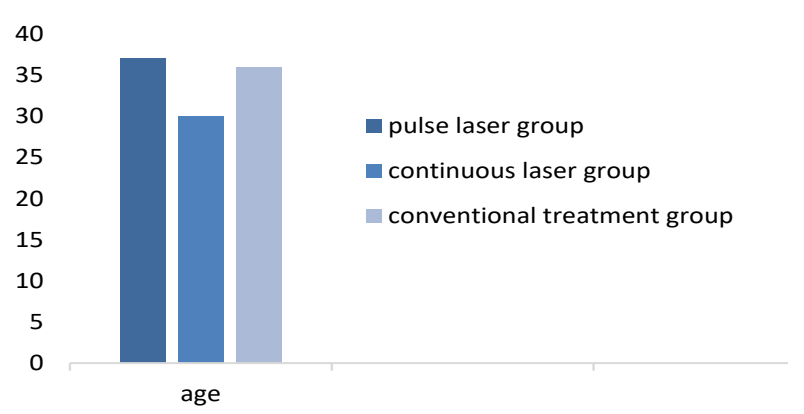

Figure 1. The Age Average of Patients in Three Different Groups.

laser, and the control group, showed the pain intensity average between groups was different, although this difference was not statistically significant $(P$ value $>$ 0.05). The comparison of pain intensity average before the intervention with time intervals of measurement pain intensity after the intervention showed in the pulsed and continuous $\mathrm{CO}_{2}$ laser groups, there is also a statistically significant difference between mean \pm observed standard error before the intervention and after the intervention including all time-intervals of measurement (immediately after the intervention, 12, 24, 48 and 72 hours after the intervention). In the control group, the difference of mean \pm standard error of pain intensity between time-intervals before the intervention and time-intervals immediately after the intervention and 72 hours after the intervention was significant. However, the difference in pain intensity average between time-intervals before the intervention and the time-intervals of 12,24 , and 40 hours after the intervention was not statistically significant (Figure 2 and Table 1).

Comparing the mean size of the ulcer at the measured time intervals in the studied groups showed the average

Table 1. Comparison of Pain Intensity Average Between Before Intervention and Time of Pain Evaluation After Intervention in Each Group

\begin{tabular}{|c|c|c|c|c|}
\hline Group & & aluation & $\begin{array}{c}\text { Mean Difference } \pm \text { Standard } \\
\text { Error }\end{array}$ & P Value \\
\hline \multirow{5}{*}{ Pulsed $\mathrm{CO}_{2}$ laser } & \multirow{5}{*}{ Before intervention } & Right after intervention & $4.6 \pm 0.68$ & 0.002 \\
\hline & & 12 hours & $4.4 \pm 0.4$ & $<0.001$ \\
\hline & & 24 hours & $4.6 \pm 0.4$ & $<0.001$ \\
\hline & & 48 hours & $3.4 \pm 0.93$ & 0.021 \\
\hline & & 72 hours & $4.4 \pm 1.08$ & 0.015 \\
\hline \multirow{5}{*}{ Continuous $\mathrm{CO}_{2}$ laser } & \multirow{5}{*}{ Before intervention } & Right after intervention & $4.6 \pm 0.98$ & 0.009 \\
\hline & & 12 hours & $3.4 \pm 0.93$ & 0.021 \\
\hline & & 24 hours & $3.4 \pm 0.93$ & 0.021 \\
\hline & & 48 hours & $4 \pm 1.05$ & 0.019 \\
\hline & & 72 hours & $4.8 \pm 0.38$ & $<0.001$ \\
\hline \multirow{5}{*}{ Usual treatment } & \multirow{5}{*}{ Before intervention } & Right after intervention & $1.8 \pm 0.49$ & 0.021 \\
\hline & & 12 hours & $2.4 \pm 1.03$ & 0.08 \\
\hline & & 24 hours & $2.6 \pm 0.98$ & 0.057 \\
\hline & & 48 hours & $3.4 \pm 1.4$ & 0.072 \\
\hline & & 72 hours & $4 \pm 1.3$ & 0.037 \\
\hline
\end{tabular}




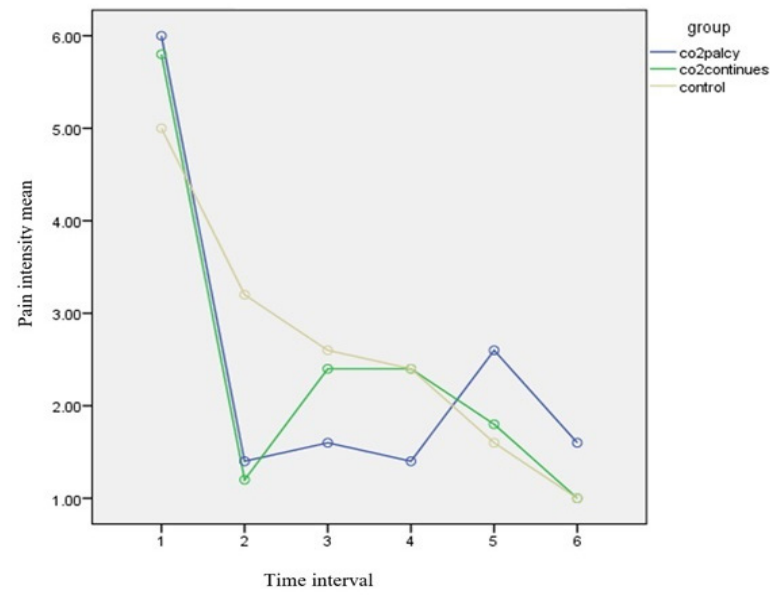

Figure 2. Comparison of the Pain Intensity Mean at Measured Time-Intervals in Three Studied Groups.

size of the ulcer on the first day in the pulsed $\mathrm{CO}_{2}$ laser group was higher than the other two groups; however, the observed difference was not statistically significant $(P$ value $=0.93)$. Comparing the mean ulcer diameter difference on the first day with the intervals of the third, fifth and seventh days in the pulsed carbon dioxide laser group revealed that the difference between the mean \pm standard error of the ulcer size on the first day and those on the third and fifth days was not significant. However, the mean difference between the first and seventh days was significant. In the continuous $\mathrm{CO}_{2}$ laser group, comparing the mean ulcer diameter difference on the first day with the third day was not significant, while the difference in the average diameter of the lesion on the first day with the fifth and seventh days was significant. In the control group, the observed results comparing the first day with the third day were meaningless, and in comparison with the first day and the fifth and seventh days they were meaningful (Figure 3 and Table 2).

\section{Discussion}

The results of this study approve a significant pain reduction in patients with RAS because of using pulsed and continuous $\mathrm{CO}_{2}$ lasers in comparison with the control

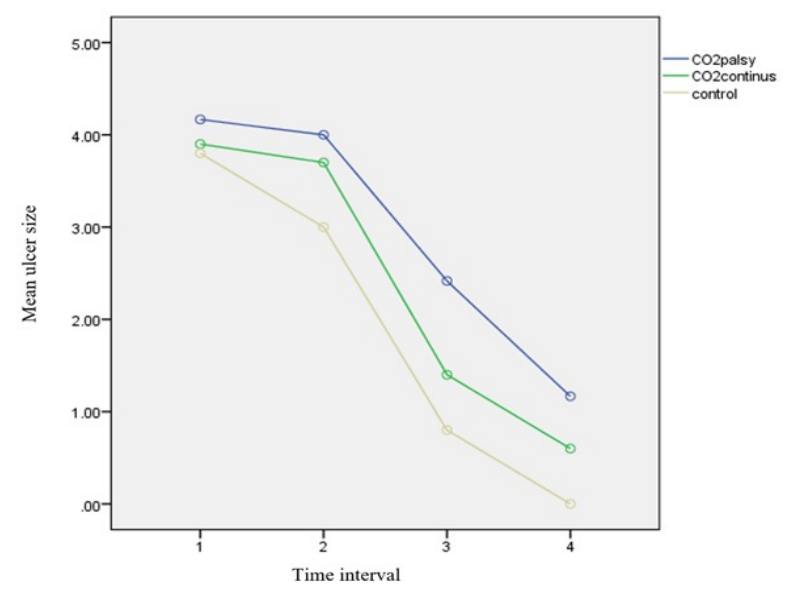

Figure 3. Comparison of the Mean Ulcer Size at Measured TimeIntervals in Three Studied Groups.

group. In various studies that have investigated the effect of lasers on aphthous ulcers in the mouth, other types of lasers have also been used. The difference in the type of lasers used is in the properties of the laser protocol (power, dose, wavelength, time of examination after the laser). In the present research, the reduction of pain in patients with recurrent oral aphthous ulcers was observed immediately after the laser and at follow-up hours, 12, 24, 40 , and 72 hours after the intervention. Zand et al showed the effect of the continuous $\mathrm{CO}_{2}$ laser with the power of 1 $\mathrm{W}$ in immediate pain reduction in follow-up hours, 4,8 , $12,24,40,72$, and 96 hours after the treatment. ${ }^{2}$

Sharon-Buller and Sela reported a case of using a $\mathrm{CO}_{2}$ laser for pain relief of recurrent aphthous lesions in a 50-year-old patient. Using a $\mathrm{CO}_{2}$ laser with a wavelength of $10600 \mathrm{~nm}$ and power of 1-1.5 W and the radiation duration of 5 seconds without topical anesthesia, she reported an immediate and prolonged pain reduction of the lesion. ${ }^{30}$ In a study by Zeini Jahromi et al, the evaluating effects of the continuous $\mathrm{CO}_{2}$ laser in comparison with the diode laser and silent laser on recurrent aphthous stomatitis indicated that patients in the group of continuous $\mathrm{CO}_{2}$ lasers with power of $2 \mathrm{~W}$ compared to patients in the other two groups experienced

Table 2. Comparison of Lesion Size Average in Time of Pain Evaluation in Each Group

\begin{tabular}{|c|c|c|c|c|c|}
\hline & & Pulsed $\mathrm{CO}_{2}$ Laser & Continuous $\mathrm{CO}_{2}$ Laser & Usual Treatment & \multirow[t]{2}{*}{$P$ Value } \\
\hline & \multicolumn{4}{|c|}{ Mean \pm Standard Deviation } & \\
\hline \multirow{7}{*}{ Lesion size } & day 1 & $4.17 \pm 1.94$ & $3.9 \pm 2.07$ & $3.8 \pm 0.84$ & 0.93 \\
\hline & day 3 & $4 \pm 1.18$ & $3.7 \pm 2.22$ & $3 \pm 1.6$ & 0.67 \\
\hline & day 5 & $2.42 \pm 2.11$ & $1.4 \pm 2.2$ & $0.8 \pm 0.84$ & 0.37 \\
\hline & day 7 & $1.17 \pm 1.81$ & $0.6 \pm 1.34$ & $0 \pm 0$ & 0.4 \\
\hline & $P$ value inside the groups & 0.02 & $<0.001$ & $<0.001$ & \\
\hline & \multicolumn{5}{|c|}{ Mean \pm Standard Error } \\
\hline & Total & $2.94 \pm 0.61$ & $2.4 \pm 0.67$ & $1.9 \pm 0.67$ & 0.53 \\
\hline
\end{tabular}


significant reduction in pain intensity. However, in terms of healing time, satisfaction, and daily dysfunction, no significant difference was observed in different groups. ${ }^{23}$ In the present study, about changing the size of RAS ulcers following the treatment in the three control groups on third, fifth and seventh days after the treatment in comparison with before the treatment, there was no significant difference. In a study by Prasad and Pai using a continuous $\mathrm{CO}_{2}$ laser with the power of $0.7 \mathrm{~W}$, lesion healing was studied on days 3 and 4 after the treatment and every two days for up to two weeks. The reduction of the erythematous halo around the aphtha and the complete ulcer removal meant to be aphtha healing. The results of this study showed the duration of ulcer healing in the laser group was shorter than that of the placebo group. ${ }^{25}$ This difference in the present study can be due to different average power and hours of follow-up and also the difference in the indicator of ulcer healing. The results of this clinical trial showed that the carbon dioxide laser, whether with continuous radiation or pulsed radiation, can be used to relieve the pain of minor aphthous lesions.

The high cost of laser therapy was one of the limitations of our study, but no cost was incurred by the patients in this regard.

\section{Ethical Considerations}

This randomized and interventional clinical trial study was done on patients referred to the clinics and offices in Urmia after obtaining permission from the Ethics Committee of Urmia University of Medical Sciences with IR.UMSU.Rec.1396.364 registration number, and written informed consent was obtained from all the patients.

\section{Conflict of Interests}

The authors declare that they have no conflict of interest.

\section{References}

1. Greenberg MS, Glick M, Ship JA. Burket's Oral Medicine. $11^{\text {th }}$ ed. Hamilton, Canada: BC Decker Inc; 2008.

2. Zand N, Ataie-Fashtami L, Djavid GE, Fateh M, Alinaghizadeh MR, Fatemi SM, et al. Relieving pain in minor aphthous stomatitis by a single session of nonthermal carbon dioxide laser irradiation. Lasers Med Sci. 2009;24(4):515-520. doi: 10.1007/s10103-008-0555-1.

3. Shashy RG, Ridley MB. Aphthous ulcers: A difficult clinical entity. Am J Otolaryngol. 2000;21(6):389-393. doi: 10.1053/ ajot.2000.18872.

4. Regezi JA, Sciubba J, Jordan RC. Oral Pathology: Clinical Pathologic Correlations. $6^{\text {th }}$ ed. St. Louis: Saunders; 2012.

5. Neville BW, D. Damm DD, Allen CM, Bouquot JE. Oral and Maxillofacial Pathology. $3^{\text {rd }}$ ed. St. Louis: Saunders; 2008.

6. Jackson K. Is Laser Therapy Effective at Relieving Pain in Adult Patients with Recurrent Aphthous Stomatitis? 2016. https://digitalcommons.pcom.edu/pa_systematic_ reviews/280.

7. Zand N, Mansouri P, Fateh M, Ataie-Fashtami L, Rezaee
Khiabanloo S, Safar F, et al. Relieving pain in oral lesions of pemphigus vulgaris using the non-ablative, non-thermal, $\mathrm{CO}_{2}$ laser therapy (NTCLT): Preliminary results of a novel approach. J Lasers Med Sci. 2017;8(1):7-12. doi: 10.15171/ jlms.2017.02.

8. Vincent SD, Lilly GE. Clinical, historic, and therapeutic features of aphthous stomatitis. Literature review and open clinical trial employing steroids. Oral Surg Oral Med Oral Pathol. 1992;74(1):79-86. doi: 10.1016/00304220(92)90219-g.

9. Ship JA. Recurrent aphthous stomatitis. An update. Oral Surg Oral Med Oral Pathol Oral Radiol Endod. 1996;81(2):141-147. doi: 10.1016/s1079-2104(96)80403-3.

10. Davatchi F, Tehrani-Banihashemi A, Jamshidi AR, ChamsDavatchi C, Gholami J, Moradi M, et al. The prevalence of oral aphthosis in a normal population in Iran: A WHOILAR COPCORD Study. Arch Iran Med. 2008;11(2):207209.

11. Alidaee MR, Taheri A, Mansoori P, Ghodsi SZ. Silver nitrate cautery in aphthous stomatitis: A randomized controlled trial. Br J Dermatol. 2005;153(3):521-525. doi: 10.1111/j.1365-2133.2005.06490.x.

12. Belenguer-Guallar I, Jiménez-Soriano Y, ClaramuntLozano A. Treatment of recurrent aphthous stomatitis. A literature review. J Clin Exp Dent. 2014;6(2):e168-74. doi: 10.4317/jced.51401.

13. Arikan OK, Birol A, Tuncez F, Erkek E, Koc C. A prospective randomized controlled trial to determine if cryotherapy can reduce the pain of patients with minor form of recurrent aphthous stomatitis. Oral Surg Oral Med Oral Pathol Oral Radiol Endod . 2006;101(1):E1-5. doi: 10.1016/j.tripleo.2005.07.035.

14. Jijin M.J, Rakaraddi M, Pai J, Jaishankar HP, Krupashankar $\mathrm{R}$, Kavitha AP, et al. Low-level laser therapy versus 5\% Amlexanox: A comparison of treatment effects in a cohort of patients with minor aphthous ulcers. Oral Surg Oral Med Oral Pathol Oral Radiol. 2016;121(3):269-273. doi: 10.1016/j.oooo.2015.11.021.

15. Stanbury R, Graham E. Systemic corticosteroid therapy: Side effects and their management. $\mathrm{Br} J$ Ophthalmol. 1998;82(6):704-708. doi: 10.1136/bjo.82.6.704.

16. Enwemeka CS. Intricacies of dose in laser phototherapy for tissue repair and pain relief. Photomed Laser Surg. 2009;27(3):387-393. doi: 10.1089/pho.2009.2503.

17. Tezel A, Kara C, Balkaya V, Orbak R. An evaluation of different treatments for recurrent aphthous stomatitis and patient perceptions: Nd:YAG laser versus medication. Photomed Laser Surg. 2009;27(1):101-106. doi: 10.1089/ pho.2008.2274.

18. Seraj B, Shahrabi M, Masoumi S, Jabbarian R, Manesh AA, Fini MB. Studying maxillary labial frenulum types and their effect on median diastema in 3-6-year-old Children in Tehran Kindergartens. World J Dent. 2019;10(2):93-97. doi: 10.5005/jp-journals-10015-1611.

19. Baghizadeh Fini M, Olyaee P, Homayouni A. The effect of low-level laser therapy on the acceleration of orthodontic tooth movement. J Lasers Med Sci. 2020;11(2):204-11. doi: 10.34172/jlms.2020.34.

20. Najeeb S, Khurshid Z, Zohaib S, Najeeb B, Qasim SB, Zafar MS. Management of recurrent aphthous 
ulcers using low-level lasers: A systematic review. Medicina (Kaunas). 2016;52(5):263-268. doi: 10.1016/j. medici.2016.07.006.

21. Anand V, Gulati M, Govila V, Anand B. Low level laser therapy in the treatment of aphthous ulcer. Indian J Dent Res. 2013;24(2):267-270. doi: 10.4103/0970-9290.116691.

22. Pavlić V, Vujić-Aleksić V, Aoki A, Nežić L. Treatment of recurrent aphthous stomatitis by laser therapy: A systematic review of the literature. Vojnosanit Pregl. 2015;72(8):722728. doi: 10.2298/vsp140410028p.

23. Zeini Jahromi N, Ghapanchi J, Pourshahidi S, Zahed M, Ebrahimi H. Clinical evaluation of high and low-level laser treatment $\left(\mathrm{CO}_{2}\right.$ vs InGaAlP diode laser) for recurrent aphthous stomatitis. J Dent (Shiraz). 2017;18(1):17-23.

24. Han M, Fang H, Li QL, Cao Y, Xia R, Zhang ZH. Effectiveness of laser therapy in the management of recurrent aphthous stomatitis: A systematic review. Scientifica (Cairo). 2016;2016:9062430. doi: 10.1155/2016/9062430.

25. Parsad S, Pai A. Assessment of immediate pain relief with laser treatment in recurrent aphthous stomatitis. Oral Surg
Oral Med Oral Pathol Oral Radiol. 2013;116(2):189-193. doi: 10.1016/j.oooo.2013.02.011.

26. Zand N, Fateh M, Ataie-Fashtami L, Djavid GE, Fatemi SM, Shirkavand A. Promoting wound healing in minor recurrent aphthous stomatitis by non-thermal, nonablative $\mathrm{CO}_{2}$ laser therapy: A pilot study. Photomed Laser Surg. 2012;30(12):719-723. doi: 10.1089/pho.2012.3301.

27. Sattayut S, Trivibulwanich J, Pipithirunkarn N, Danvirutai N. A clinical efficacy of using $\mathrm{CO}_{2}$ laser irradiating to transparent gel on aphthous stomatitis patients. Laser Ther. 2013;22(4):283-289. doi: 10.5978/islsm.13-OP-24.

28. Rezvaninezhad RS, Navabi N, Atai Z, Shahravan A. The effect $\mathrm{CO}_{2}$ laser on reducing pain associated with aphthous stomatitis. J Babol Univ Med Sci. 2016;18(10):20-25.

29. Colvard M, Kuo P. Managing aphthous ulcers: Laser treatment applied. J Am Dent Assoc. 1991;122(6):51-53. doi: 10.1016/s0002-8177(91)26017-1.

30. Sharon-Buller A, Sela M. $\mathrm{CO}_{2}$-laser treatment of ulcerative lesions. Oral Surg Oral Med Oral Pathol Oral Radiol Endod. 2004;97(3):332-334. doi: 10.1016/j.tripleo.2003.11.012. 